\title{
Configurational energy and the formation of mixed flowing/ powder snow and ice avalanches
}

\author{
Perry BARTELT, Othmar BUSER, Cesar VERA VALERO, Yves BÜHLER \\ WSL Institute for Snow and Avalanche Research SLF, Davos-Dorf, Switzerland \\ Correspondence: Perry Bartelt <bartelt@slf.ch>
}

\begin{abstract}
A long-standing problem in avalanche dynamics is to model the flow of a mixed flowing/ powder avalanche. Here we use the thermodynamic concept of configurational energy to describe the blow-out of air from the avalanche core. Configurational energy is the mean potential energy associated with the location of snow and ice particles in the avalanche core. As such, configurational energy determines the avalanche flow density. Expansion of the particle ensemble reduces the flow density and leads to the intake of air. Compression of the particle ensemble causes the blow-out of the intaken air, now laden with ice dust. Once formed, the cloud moves independently of the flowing avalanche with the initial momentum acquired in the core. Configurational energy changes in the avalanche core are therefore intimately related to the formation of the powder suspension cloud. In this paper we use the concept of configurational energy to predict the mass of air taken into and blown out of the core. This requires calculating the dispersive pressure arising from random particle movements and configuration changes related to the expansion and collapse of the flowing particle ensemble. The ice avalanche that struck the Everest base camp on 25 April 2015 is simulated using the proposed concept.
\end{abstract}

KEYWORDS: avalanches

\section{INTRODUCTION}

Recent avalanche disasters in Nepal have highlighted the need for avalanche dynamics models to predict the motion of mixed flowing/powder avalanches (CBS News, 2015; Jenkins, 2015; Wilkinson, 2015). Mixed flowing/powder avalanches are characterized by a fast-moving core of heavy ice/snow particles and a powder suspension cloud consisting of fine ice dust (Figs 1 and 2). These avalanches are especially dangerous because they reach high velocities and long runout distances, especially in the cold, steep terrain of the Himalayan mountain range. The area inundated by the avalanche is difficult to predict because the powder cloud can decouple from the avalanche core and move independently, reaching distances well beyond the reach of the dense core.

Models to describe the formation and movement of mixed snow and ice avalanches were proposed by Russian scientists in the former Soviet Union (Bozhinskiy and Losev, 1998). Russian scientists were able to track the movement of mixed avalanches using simple photogrammetric methods and three pressure masts strategically placed in the runout zone of their observation site in the Caucasus (Grigoryan and others, 1982). With this unique (and unduplicated) setup, the Russians were able to measure the evolution in time and space of powder cloud velocity and pressure (Sukhanov and Kholobayev, 1982). The models derived from the experimental observations divide avalanche flow into two layers: the flowing core (denoted $\Phi$ ) and the powder suspension cloud (denoted $\Pi$ ) (Fig. 3; Bozhinskiy and Losev, 1998). The mass in the avalanche core consists of heavier snow particles that cannot be suspended in the cloud. Empirical relationships were derived to describe the mass and momentum exchanges between the core and powder cloud (Nazarov, 1990). The fundamental contribution of Russian scientists, however, was to treat the dynamics of the powder cloud as an inertial flow, independent of the core, but with initial conditions given by the core. The cloud velocity attenuates by aerodynamic drag.

The mathematical concepts invoked by the Russians provide the first step in the direction of a practical mixed flowing/powder avalanche model. These concepts, however, are physically limited by the assumption of a constant flow density in the avalanche core $\Phi$. The ice dust suspended in the powder cloud functions as a tracer for the air that is expelled from the core $\Phi$. To create the powder cloud, air must be taken in by the core, particularly at the avalanche front, and loaded with ice dust. As the core moves rapidly in the slope-parallel direction, random particle movements cause the core to expand, leading to open spaces at the avalanche front which facilitate the consumption of air. At this point the avalanche core contains a disperse and wild mixture of snow-cover

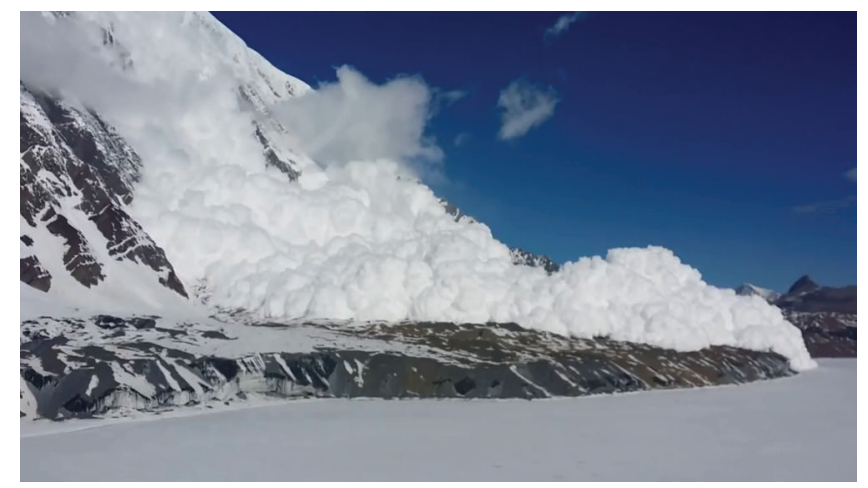

Fig. 1. The powder cloud of a large mixed flowing/powder snow avalanche crosses Tilicho lake in the Annapurna, Nepal. The cloud is $\sim 100 \mathrm{~m}$ high. Note the steep acceleration zone. The avalanche travelled across the frozen $3 \mathrm{~km}$ long lake, reaching the opposite shore in $<60 \mathrm{~s}$. This mixed flowing/powder avalanche entrained little additional mass. 


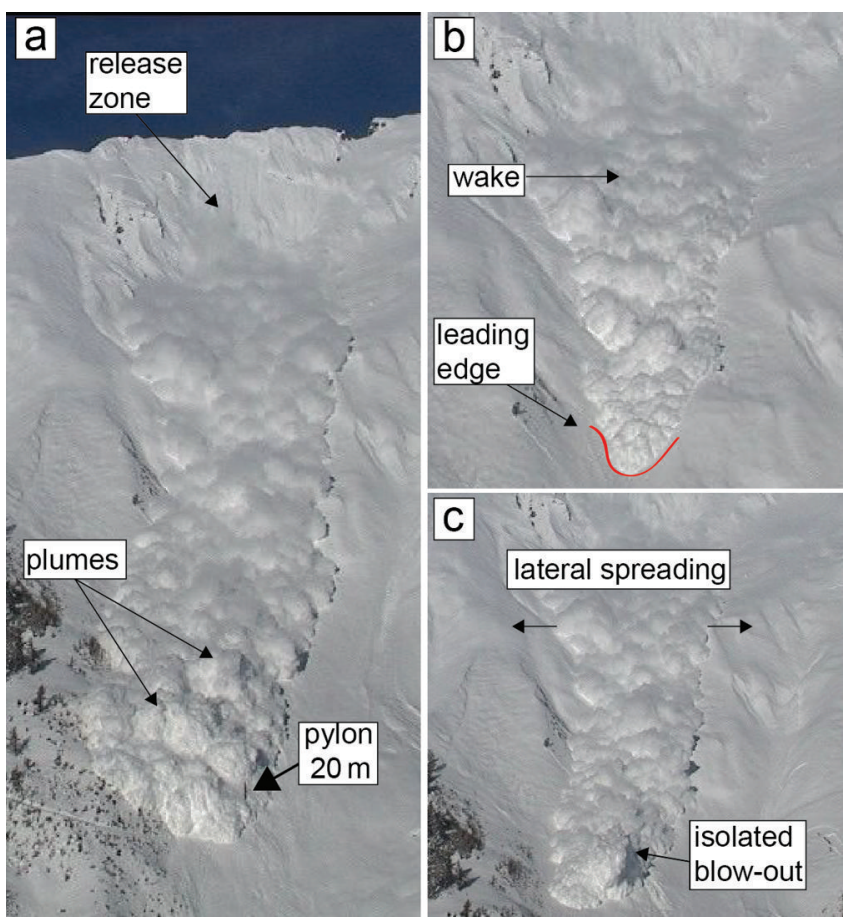

Fig. 2. Mixed flowing/powder avalanches artificially released at the Vallée de la Sionne test site. (a) Avalanche No. 509 of 7 February 2003 strikes the measurement pylon. Note the blow-out plumes at the front of the avalanche. Initial powder cloud heights are between 5 and $10 \mathrm{~m}$; the plumes grow to $40-50 \mathrm{~m}$ before dispersing. (b) Avalanche No. 628 released on 19 January 2004. The pictures were taken $\sim 50 \mathrm{~s}$ after release. The leading edge of the avalanche is moving at a speed of $60 \mathrm{~m} \mathrm{~s}^{-1}$ whereas the wake is almost stationary. Large velocity gradients exist between the front and tail of the cloud. (c) A large isolated blow-out at the avalanche front. The speed at which the powder cloud spreads in the lateral direction is smaller than the front speed of the avalanche. If density gradients controlled the motion of the cloud, it would spread in all directions at the same speed.

fragments, snow/ice particles and ice dust. It is not possible to define the boundary between the core $\Phi$ and cloud $\Pi$. The blow-out phase begins as heavier particles settle, displacing the interstitial air. Because the avalanche is moving forward, the easiest escape for the ice dust mixture is upwards. Sudden terrain changes and channel constrictions that cause the core to densify will likewise expel air. As the core compresses, the ice dust mixture is ejaculated in plume-like formations which give powder avalanches their distinctive lobe-cleft structure (Bozhinskiy and Sukhanov, 1998; Bartelt and others, 2013; Fig. 2). The ice dust cloud moves forward with the momentum acquired in the avalanche core (Sukhanov and Kholobayev, 1982). Plume formation is likewise a segregation process, as it results in the separation of the ice dust from the heavier snow particles. The driving idea behind all two-layer avalanche models is that a blow-out process separates the core $\Phi$, containing heavier snow particles, from the ice dust that remains suspended in the cloud $\Pi$. When the segregation process is complete, it is possible to distinguish between the two layers $\Phi$ and $\Pi$.

In this paper we develop a mathematical model to describe the mixing, air blow- out and segregation processes involved in the formation of mixed flowing/powder snow avalanches. This requires a method to account for streamwise density variations in the avalanche core. We account for the density variations by tracking the energy associated with random particle movements and locations (Buser and Bartelt, 2015). This energy is divided into kinetic and potential parts and accounts for the changing configuration of the heavy particles. This idea is used in thermodynamic theories of granular materials (Luca and others, 2004). We introduce the concept of configurational energy within the framework of computationally efficient two-layer avalanche dynamics models. Unlike Russian formulations, we therefore consider streamwise density variations which allow us to define the necessary mass and momentum exchanges involved in the compression of the core $\Phi$ and therefore formation of the powder cloud $\Pi$.

\section{AVALANCHE MASS AND DENSITY}

To model mixed flowing/powder avalanches we divide the moving body into representative segments or volumes $V_{\Phi}$ and $V_{\Pi}$ (Figs 3 and 4 ). The volumes are defined by the (constant) basal area $A$ and flow heights of the avalanche core $h_{\Phi}$ and cloud $h_{\Pi}$. Both heights are defined with respect to the base of the core. The core and cloud are initially coextensive. When the cloud detaches $\left(h_{\Phi}=0\right)$, the height of the cloud is measured from the gliding surface of the avalanche. The volumes are fixed to a particular location

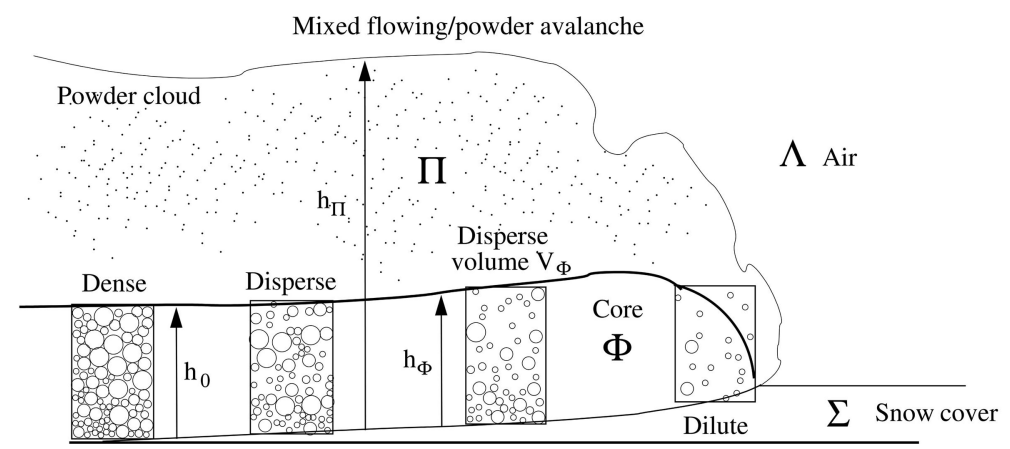

Fig. 3. Schematic diagram of a mixed flowing/powder avalanche system. A mixed flowing/powder avalanche contains four basic components: the avalanche core $\Phi$, the cloud $\Pi$, the snow cover $\Sigma$ and the outside air $\Lambda$. The volume $V_{\Phi}$ is defined by the flow height $h_{\Phi}$ and the basal area $A, V_{\Phi}=h_{\Phi} A$. The height of the co-volume $V_{0}$ is $h_{0}$. The mass per unit area is denoted $M_{\Phi}$. The height of the cloud is $h_{\Pi}$. The heights of the core and the cloud are defined with respect to the base of the core. For the numerical treatment the volumes are at fixed positions, i.e. they are not moving with the avalanche. The avalanche is flowing through the volumes. 

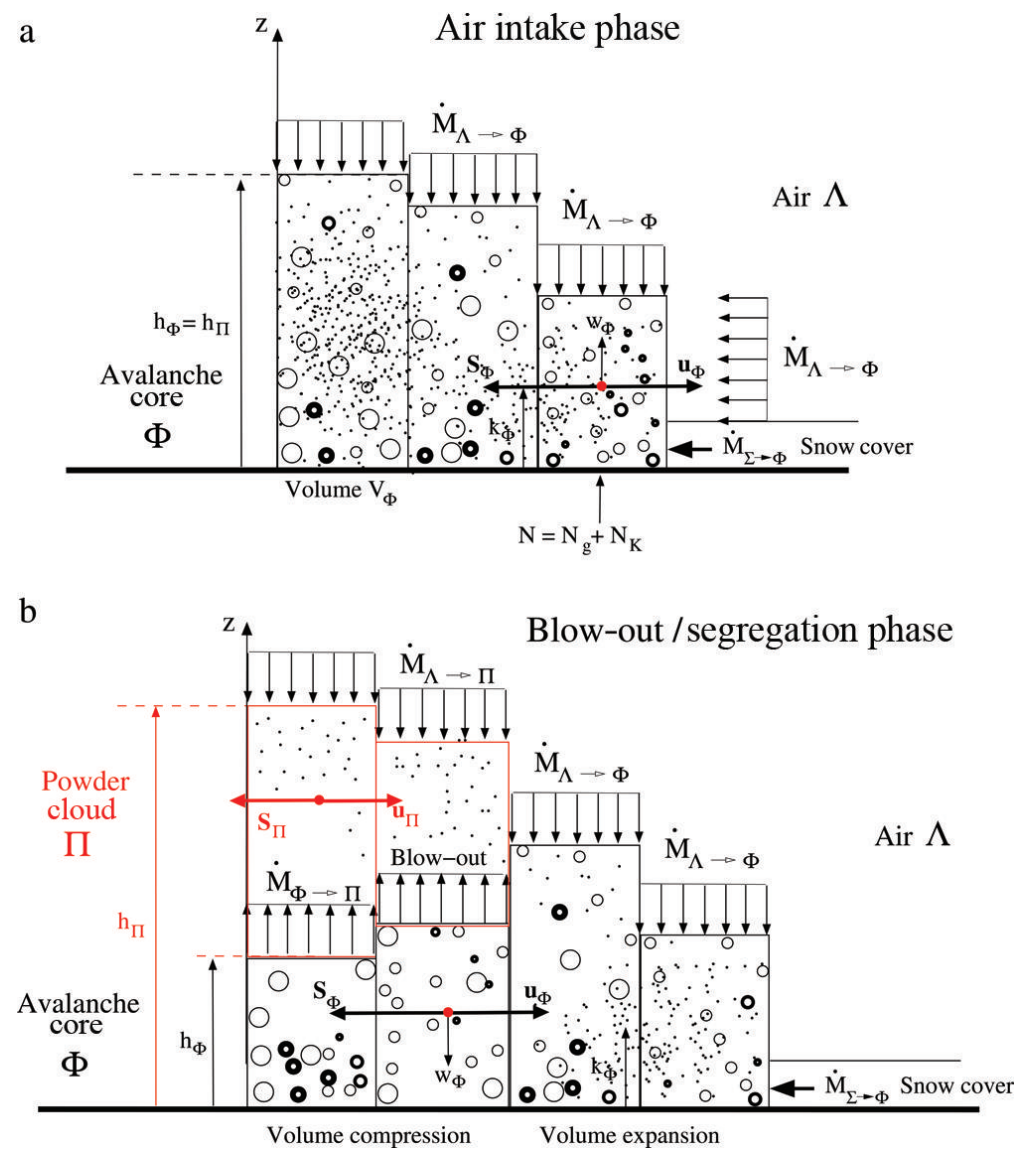

Fig. 4. Cross section of a two-layer mixed flowing/powder avalanche model showing the core $\Phi$ and cloud $\Pi$. The velocities $\mathbf{u}_{\Phi}$ and $\mathbf{u}_{\Pi}$ are defined in the direction parallel to the ground. The expansion velocity of the core $w_{\Phi}$ is defined perpendicular to the ground. The core entrains the snow cover $\Sigma$ at the rate $\dot{M}_{\Sigma \rightarrow \Phi}$ and ambient air $\Lambda$ at the rate $\dot{M}_{\Lambda \rightarrow \Phi}$. (a) Expansion of the core results in the air intake phase. The air is loaded with ice dust. (b) Compression of the core leads to the blow-out of air and ice dust.

(Eulerian formulation), and mass flows through the volume with mean velocities $\mathbf{u}_{\Phi}=\left(u_{\Phi}, v_{\Phi}\right)^{T}$ and $\mathbf{u}_{\Pi}=\left(u_{\Pi}, v_{\Pi}\right)^{T}$ (Figs 3 and 4$)$. The velocity vectors are defined in the slopeparallel direction (Fig.4). The velocities $\mathbf{u}_{\Phi}$ and $\mathbf{u}_{\Pi}$ are mean velocities of each layer as we do not take into account velocity variations in the $z$-direction.

The volume $V_{\Phi}$ contains the solid particle mass $M_{\Phi}$, and $V_{\Pi}$ the ice dust mass $M_{\Pi}$ including the mass of air. The mass is defined per unit area $A$. The configuration of the particle mass within the avalanche core can vary (Fig. 3). This gives rise to different density distributions in the core. For example, the particle mass can collapse into a solid volume with height $h_{0}$ measured with reference to the density of a random packing density $\rho_{0}$ :

$$
M_{\Phi}=\rho_{0} h_{0} .
$$

Because the height $h_{0}$ represents the height of a dry deposition pile of snow and ice particles it is given a special designation, the co-volume height. Another option is to assume the particle mass is distributed uniformly over the flow volume, in which case

$$
M_{\Phi}=\rho_{\Phi} h_{\Phi} \quad \text { and } \quad M_{\Pi}=\rho_{\Pi} h_{\Pi} .
$$

The mean densities of the core and cloud are thus $\rho_{\Phi}$ and $\rho_{\Pi \text {, }}$ respectively. The heights $h_{0}$ and $h_{\Phi}$ represent two different flow configurations that the avalanche core may assume with the same mass. The height $h_{0}$ is typically encountered when the avalanche has settled in the deposition zone, the random packing density being close to the deposition density of the granular phase. That is, it is encountered when the avalanche core is at rest.

\section{DISPERSIVE PRESSURE}

To change the location of the snow and ice material from the co-volume configuration $h_{0}$ to the flow configuration $h_{\Phi}$ requires mechanical work. The only source of work (energy) in the avalanche is the gravitational potential. Gravitational potential energy is converted into kinetic energy in the slope-parallel direction and heat. It is the interaction of the particles that converts the kinetic energy of the mean slopeparallel flow into configurational work: when the particles are moving downwards they encounter the slope roughness. Snow and ice particles hit the ground and are reflected upwards back into the flow; local contact pressures are large. However, as we consider the total ensemble of particles in the volume $V_{\Phi}$, the net effect of all the particle interactions is to define the movement of the center-of-mass in the slope-perpendicular $z$-direction. We denote the $z$-position of the center-of-mass $k_{\Phi}$ and the slope-perpendicular acceleration $\ddot{k}_{\Phi}$ (Fig. 4). If the particle interactions generate pressures to change the location of the center-ofmass, there must be a corresponding reactive pressure at the basal boundary. This is the dispersive pressure $N_{K}$ (see Fig. 4a; Bagnold, 1954). This pressure is given by the mass $M_{\Phi}$ in the avalanche core and the acceleration $\ddot{k}_{\Phi}$,

$$
N_{K}=M_{\Phi} \ddot{k}_{\Phi} \text {. }
$$


The total reaction at the basal boundary $N$ is the sum of the weight $N_{g}$,

$$
N_{g}=M_{\Phi} g_{z}
$$

and $N_{K}$,

$$
N=N_{g}+N_{K}
$$

The gravity component in the slope-perpendicular direction is denoted $g_{z}$. The sum of the accelerations $g_{z}$ and $\ddot{k}_{\Phi}$ is denoted $g^{\prime}$. Because we include the pressure $N_{K}$, the pressure distribution is no longer hydrostatic. Basal pressure measurements cannot distinguish between a change in mass $M_{\Phi}$ or a change in the location of the center-of-mass $k_{\Phi}$ because the total normal force $N$ is the sum of $N_{g}$ and $N_{K}$ (Platzer and others, 2007). It is therefore difficult to determine the dispersive pressure experimentally. A connection to experimental measurements can nonetheless be made by noting that the time rate of change of the normal pressure $\dot{N}$ is

$$
\dot{N}=M_{\Phi} \dddot{k}_{\Phi}
$$

when the mass is constant in the volume $V_{\Phi}$. Because gravitational acceleration $g_{z}$ is constant, it disappears from the time derivative. The quantity $\dddot{k}_{\Phi}$ is the boundary jerk.

\section{FREE MECHANICAL ENERGY}

Shearing is the immediate source of the energy needed to change the configuration of the avalanche core. The work rate $\dot{W}_{f}$ of the shear in the avalanche core is

$$
\dot{W}_{f}=\mathbf{S}_{\Phi} \cdot \mathbf{u}_{\Phi},
$$

where $\mathbf{S}_{\Phi}$ is the shear traction at the base of the avalanche core. The work rate $\dot{W}_{f}$ represents the total work done per unit time $\left(\mathrm{W} \mathrm{m}^{-2}\right)$. In the standard Voellmy model the shear work is dissipated at the rate $\dot{Q}$ to heat energy $E$. In a model with configurational changes the shear work produces not only heat $E$, but also free mechanical energy $R$ which is divided into kinetic energy $R_{K}$ and potential (configurational) energy $R_{V}$ (Bartelt and others, 2006; Buser and Bartelt, 2015),

$$
R=R_{K}+R_{V}
$$

$R_{K}$ is the kinetic energy associated with all particle movements different from the mean velocity of the flow. When particles hit the ground, they have some velocity component in the slope-perpendicular direction; that is, different from the mean downslope direction of the flow. The kinetic energy associated with the slope-perpendicular movement is contained in $R_{K} . R_{V}$ is the potential energy of the centerof-mass of the particles. This energy is defined with respect to the bottom of the avalanche

$$
R_{V}=\frac{1}{2} M_{\Phi} g^{\prime}\left[h_{\Phi}\right]
$$

One part of the free energy is therefore true kinetic energy $R_{K}$, while the remaining part $R_{V}$ describes the changed location of the particles. The production rates of $R_{K}$ and $R_{V}$ in the volume are denoted $\dot{P}_{K}$ and $\dot{P}_{V}$. Thus, balance equations for the free mechanical energy can be written,

$$
\begin{aligned}
& \frac{\mathrm{D}\left(R_{K} h_{\Phi}\right)}{\mathrm{D} t}=\dot{P}_{K} h_{\Phi}, \\
& \frac{\mathrm{D}\left(R_{V} h_{\Phi}\right)}{\mathrm{D} t}=\dot{P}_{V} h_{\Phi}
\end{aligned}
$$

and

$$
\frac{\mathrm{D}\left(R h_{\Phi}\right)}{\mathrm{D} t}=\dot{P} h_{\Phi}=\dot{P}_{K} h_{\Phi}+\dot{P}_{V} h_{\Phi}
$$

These equations are written using the material derivative notation to indicate that we must also consider the convective transport of $R_{K}$ and $R_{V}$. In configurational models, the shear work is not all dissipated to heat, but used also to produce free mechanical energy,

$$
\dot{W}_{f}=\dot{Q} h_{\Phi}+\dot{P} h_{\Phi}
$$

Different relations can be used to separate the heat dissipation from the production of free mechanical energy. In snow avalanche models the following splitting is applied (Bartelt and others, 2006):

$$
\begin{gathered}
\frac{\mathrm{D}\left(R h_{\Phi}\right)}{\mathrm{D} t}=\dot{P} h_{\Phi}=\alpha \dot{W}_{f}^{x y}-\beta_{K} R_{K} h_{\Phi} \\
\frac{\mathrm{D}\left(E h_{\Phi}\right)}{\mathrm{D} t}=\dot{Q} h_{\Phi}=(1-\alpha) \dot{W}_{f}+\beta_{K} R_{K} h_{\Phi} .
\end{gathered}
$$

The parameter $\alpha$ is the splitting parameter, defining the partitioning of the frictional work rate into the production of free mechanical energy $\dot{P}$ and heat energy $\dot{Q}$. The parameter $\beta_{K}$ defines the dissipation of free kinetic energy of the granular solid by collisions, rubbing, abrasion, etc. It can be shown that this procedure is energy-conserving (Buser and Bartelt, 2015).

The sum of $\dot{P}_{K}$ (change in random kinetic energy) and $\dot{P}_{V}$ (change in configuration energy) therefore defines the production of total mechanical free energy $\dot{P}$ in the volume $V_{\Phi}$

$$
\dot{P}=\dot{P}_{K}+\dot{P}_{V}
$$

We define the fraction of mechanical energy $\dot{P}_{V}$ with respect to the total production of free energy $\dot{P}$ as the dimensionless coefficient $\gamma$ :

$$
\dot{P}_{V}=\gamma \dot{P} \quad \text { and } \quad \dot{P}_{K}=(1-\gamma) \dot{P} .
$$

The coefficient $\gamma$ resembles the coefficient of thermal expansion since it describes how a material changes its volume in response to a temperature change, in this case the granular temperature. It is physically constrained between the values $0<\gamma<1$. Values $\gamma=0$ or $\gamma=1$ are physically impossible because $R_{V}$ and $R_{K}$ coexist.

\section{AIR INTAKE/ICE-DUST BLOW-OUT}

The problem now is to relate the configurational energy production $\dot{P}_{V}$ to the change in the location of the center-ofmass $k_{\Phi}$. This determines the volume of air that can be taken in by the avalanche core as well as the amount of ice dust that can be blown out of the core. We emphasize that $\dot{P}_{V}$ is simply some fraction of the shear power $\dot{W}_{f}$ that is transformed into potential energy. The work done by $\dot{P}_{V}$ is used to change the volume $V_{\Phi}$ of the avalanche. Therefore,

$$
\frac{\mathrm{d}\left(N V_{\Phi}\right)}{\mathrm{d} t} \equiv \dot{P}_{V} V_{\Phi},
$$




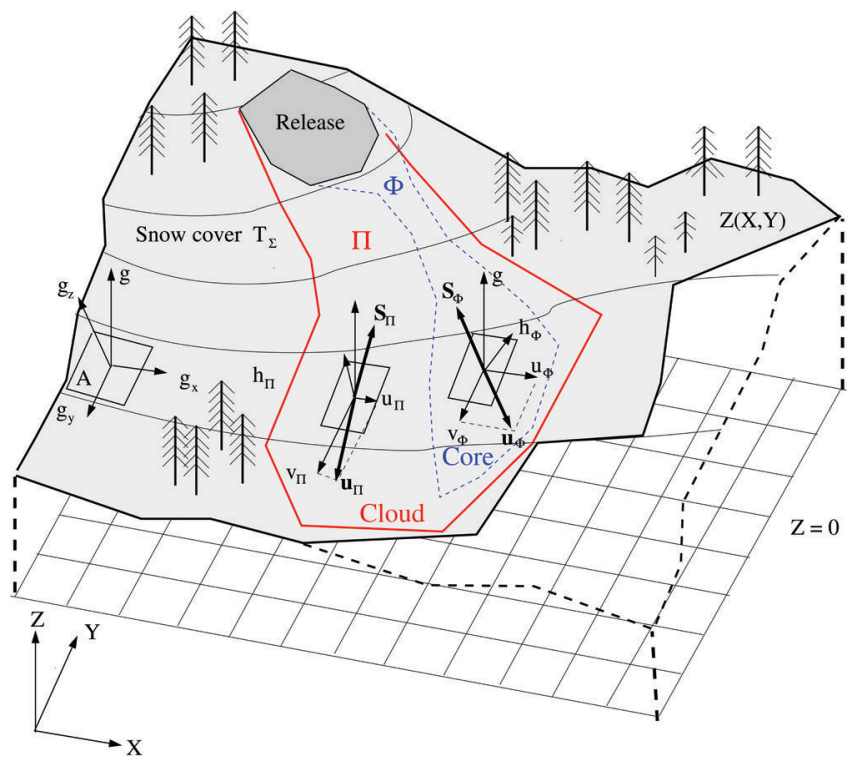

Fig. 5. A segment of the mountain terrain which is defined with plane elements in a global $X Y Z$ coordinate system. Each plane element has area $A$ and local coordinate system $x y z$. The local coordinate system defines the gravitational accelerations $g_{x}, g_{y}$ and $g_{z}$. Two-layer models consider the motion of the avalanche core $\Phi$ with slope-parallel velocity $\mathbf{u}_{\Phi}$ and the motion of the cloud $\Pi$ with velocity $\mathbf{u}_{\Pi}$. The shear stress $\mathbf{S}_{\Phi}$ opposes the flow. The flow height of the core layer is $h_{\Phi}$ and the flow height of the powder layer is $h_{\Pi}$.

or

$$
\dot{N}+N \frac{\dot{V}_{\Phi}}{V_{\Phi}}=\gamma \dot{P}=\dot{P}_{V}
$$

or, by substitution of Eqns (3-6),

$$
M_{\Phi} \dddot{k}_{\Phi}+M_{\Phi}\left[g_{z}+\ddot{k}_{\Phi}\right] \frac{\dot{k}_{\Phi}}{k_{\Phi}}=\dot{P}_{V} .
$$

For the integration of the dispersive pressure and boundary jerk into numerical simulation programs, this equation can be conveniently written into a series of three first-order differential equations, namely

$$
\begin{gathered}
\frac{\mathrm{D} k_{\Phi}}{\mathrm{D} t}=w_{\Phi} \\
\frac{\mathrm{D}\left(M_{\Phi} w_{\Phi}\right)}{\mathrm{D} t}=N_{K}, \\
\frac{\mathrm{D} N_{K}}{\mathrm{D} t}+\left[\frac{N}{k_{\Phi}}\right] w_{\Phi}=\dot{P}_{V} .
\end{gathered}
$$

These first-order equations allow us to include the transport of $k_{\Phi}$ (core height), $M_{\Phi} W_{\Phi}$ (momentum in the z-direction) and $N_{K}$ (dispersive pressure). Moreover, the jerk associated with the convective mass transport must be and is considered in the depth-averaged, two-layer avalanche model.

The formation of the powder cloud $\Pi$ is modelled as a

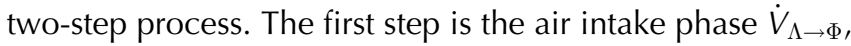
where $\Lambda$ represents the air surrounding the avalanche and contains no ice dust (see Fig. 3). The entrainment rate is given by the growth rate of the height $h_{\Phi}$, which is defined by the location of the highest solid particle in the flow column (Fig. 4a). As we assume a uniform density profile in the avalanche core, the growth rate is twice the vertical velocity $w_{\Phi}$,

$$
\dot{V}_{\Lambda \rightarrow \Phi}=2 w_{\Phi} \text { for } \quad w_{\Phi}>0 .
$$

Here we adopt the Russian notation of using $\dot{V}_{\Lambda \rightarrow \Phi}$ to signify a change in height (dimension $\mathrm{m} \mathrm{s}^{-1}$ ). Different assumptions regarding the density distribution will lead to different entrainment rates. The assumption of a homogeneous density distribution agrees well with measured core heights at the Vallée de la Sionne (Switzerland) test site (Buser and Bartelt, 2015).

Ice dust is mixed with the pure air to create a higherdensity interstitial fluid. The density of the ice dust mixture is $\rho_{\Pi_{0}}$. Moreover, we assume a constant density $\rho_{\Pi_{0}}$. This is the initial density of the powder cloud in the avalanche core. During the plume blow-out, more air is entrained which will dilute this mixture. However, the consumption of air and the mixing with ice dust during the first phase leads to the initial growth rate of the powder cloud inside the core,

$$
\dot{M}_{\Lambda \rightarrow \Phi}=\rho_{\Pi_{0}} \dot{V}_{\Lambda \rightarrow \Phi}, \text { for } \quad w_{\Phi}>0 .
$$

At this point it is not possible to distinguish the core and the cloud. Air intake at the avalanche front is implicitly assumed in the core equations and can be calculated by determining the slope-perpendicular core expansion velocity $w_{\Phi}$. Core expansion is controlled by the dispersive pressure, which is a damped 'harmonic' process (Buser and Bartelt, 2011). The front of the avalanche is special in that it can easily entrain ambient air, which is not possible for volume elements towards the tail of the avalanche. We do not consider the energy loss required to acclerate the air in the avalanche core.

In the second phase of the powder cloud formation, the air consumed at the avalanche front is blown out of the core (Fig. 4b). Blow-out is related to the downward (negative) z-velocity of the core flow column,

$$
\dot{V}_{\Phi \rightarrow \Pi}=2 w_{\Phi}, \quad \text { for } \quad w_{\Phi} \leq 0 .
$$

The mass growth rate of the cloud is therefore

$$
\dot{M}_{\Phi \rightarrow \Pi}=\rho_{\Pi_{0}} \dot{V}_{\Phi \rightarrow \Pi,} \text { for } \quad w_{\Phi} \leq 0 .
$$

Because the larger particles and snow fragments cannot be suspended in the cloud, they return to the flow, collapsing the core and squeezing out the ice dust mixture. As we assume that the ice dust is now moving with the speed of the core, it is imparted with an initial momentum in the 
downslope direction. We do not consider the reactive normal stress due to the $z$-direction acceleration of the ice dust/air mixture. Furthermore, we do not consider the additional weight of the suspended mixture on the core.

\section{TWO-LAYER MODEL EQUATIONS}

Two-layer avalanche model equations have the general form

$$
\begin{gathered}
\frac{\partial \mathbf{U}_{\Phi}}{\partial t}+\frac{\partial \boldsymbol{\Phi}_{x}}{\partial x}+\frac{\partial \boldsymbol{\Phi}_{y}}{\partial y}=\mathbf{G}_{\Phi,} \\
\frac{\partial U_{\Pi}}{\partial t}+\frac{\partial \boldsymbol{\Pi}_{x}}{\partial x}+\frac{\partial \boldsymbol{\Pi}_{y}}{\partial y}=G_{\Pi} .
\end{gathered}
$$

The mathematical description of mountain terrain is defined using a horizontal $X-Y$ coordinate system. A local surface $(x, y, z)$ coordinate system with the directions $x$ and $y$ parallel to the metric geographic coordinates $X$ and $Y$ is used (Fig. 5). Gravitational acceleration is decomposed into three gravitational components, $\mathbf{g}=\left(g_{x}, g_{y}, g_{z}\right)$. The vectors $\mathbf{U}_{\Phi}$ and $\mathbf{U}_{\Pi}$ contain the avalanche state variables for the core $\Phi$ and suspension cloud $\Pi$ defined with respect to the local $(x, y, z)$ coordinate system. For the core, we use the model equations of Buser and Bartelt (2015)

$$
\mathbf{U}_{\Phi}=\left(M_{\Phi}, M_{\Phi} u_{\Phi}, M_{\Phi} v_{\Phi}, R h_{\Phi}, h_{\Phi}, M_{\Phi} W_{\Phi}, N_{K}\right)^{T}
$$

and for the cloud, we apply the model equations proposed by Russian researchers Nazarov (1990) and Bozhinskiy and Losev (1998)

$$
\mathbf{U}_{\Pi}=\left(M_{\Pi}, M_{\Pi} u_{\Pi}, M_{\Pi} v_{\Pi}, h_{\Pi}\right)^{T} .
$$

The flux components for the core $\left(\boldsymbol{\Phi}_{x,} \boldsymbol{\Phi}_{y}\right)$ are

$$
\boldsymbol{\Phi}_{X}=\left(\begin{array}{c}
M_{\Phi} u_{\Phi} \\
M_{\Phi} u_{\Phi}^{2}+\frac{1}{2} M_{\Phi} g^{\prime} h_{\Phi} \\
M_{\Phi} u_{\Phi} v_{\Phi} \\
R h_{\Phi} u_{\Phi} \\
h_{\Phi} u_{\Phi} \\
M_{\Phi} W_{\Phi} u_{\Phi} \\
N_{K} u_{\Phi}
\end{array}\right), \boldsymbol{\Phi}_{y}=\left(\begin{array}{c}
M_{\Phi} v_{\Phi} \\
M_{\Phi} u_{\Phi} v_{\Phi} \\
M_{\Phi} v_{\Phi}^{2}+\frac{1}{2} M_{\Phi} g^{\prime} h_{\Phi} \\
R h_{\Phi} v_{\Phi} \\
h_{\Phi} v_{\Phi} \\
M_{\Phi} W_{\Phi} v_{\Phi} \\
N_{K} v_{\Phi}
\end{array}\right)
$$

and the flux components of the cloud $\left(\boldsymbol{\Pi}_{x}, \boldsymbol{\Pi}_{y}\right)$ are

$$
\Pi_{x}=\left(\begin{array}{c}
M_{\Pi} u_{\Pi} \\
M_{\Pi} u_{\Pi}^{2}+\frac{1}{2} M_{\Pi} g_{z} h_{\Pi} \\
M_{\Pi} u_{\Pi} v_{\Pi} \\
h_{\Pi} u_{\Pi}
\end{array}\right), \Pi_{y}=\left(\begin{array}{c}
M_{\Pi} v_{\Pi} \\
M_{\Pi} u_{\Pi} v_{\Pi} \\
M_{\Pi} v_{\Pi}^{2}+\frac{1}{2} M_{\Pi} g_{z} h_{\Pi} \\
h_{\Pi} v_{\Pi}
\end{array}\right) .
$$

The two driving terms $\mathbf{G}_{\Phi}$ and $\mathbf{G}_{\Pi}$ of the two-layer model system are given by

$$
\mathbf{G}_{\Phi}=\left(\begin{array}{c}
\dot{M}_{\Sigma \rightarrow \Phi}-\dot{M}_{\Phi \rightarrow \Pi} \\
G_{x}-S_{\Phi x}-\dot{M}_{\Phi \rightarrow \Pi} u_{\Phi} \\
G_{y}-S_{\Phi y}-\dot{M}_{\Phi \rightarrow \Pi} V_{\Phi} \\
\alpha\left(\mathbf{S}_{\Phi} \cdot \mathbf{u}_{\Phi}\right)-\beta R_{K} \\
W_{\Phi} \\
N_{K} \\
2 \gamma \dot{P}-2 N w_{\Phi} / h_{\Phi}
\end{array}\right)
$$

and

$$
\mathbf{G}_{\Pi}=\left(\begin{array}{c}
\dot{M}_{\Phi \rightarrow \Pi}+\dot{M}_{\Lambda \rightarrow \Pi} \\
\dot{M}_{\Phi \rightarrow \Pi} u_{\Phi}-S_{\Pi x} \\
\dot{M}_{\Phi \rightarrow \Pi} v_{\Phi}-S_{\Pi y} \\
\dot{V}_{\Phi \rightarrow \Pi}+\dot{V}_{\Lambda \rightarrow \Pi}
\end{array}\right)
$$

The core of the avalanche is driven by the gravitational acceleration in the tangential directions $\mathbf{G}=\left(G_{x}, G_{y}\right)=$ $\left(M_{\Phi} g_{x}, M_{\Phi} g_{y}\right)$. In this model the cloud can disengage from the core and move independently. The runout distance of the cloud can be long and extend well beyond the stopping distance of the core.

For shearing in the core $\mathbf{S}_{\Phi}=\left(S_{\Phi x}, S_{\Phi y}\right)$ we use a Voellmy-type ansatz

$$
\mathbf{S}_{\Phi}=\frac{\mathbf{u}_{\Phi}}{\left\|\mathbf{u}_{\Phi}\right\|}\left[\mu\left(R_{V}\right) N+\rho_{0} g \frac{\left\|\mathbf{u}_{\Phi}\right\|^{2}}{\xi\left(R_{V}\right)}\right] .
$$

The two friction parameters are Coulomb friction $\mu$ and turbulent friction $\xi$. Drag on the powder cloud, $\mathbf{S}_{\Pi}=\left(S_{\Pi x}, S_{\Pi y}\right)$, is given by a velocity-squared-type law

$$
\mathbf{S}_{\Pi}=\frac{\mathbf{u}_{\Pi}}{\left\|\mathbf{u}_{\Pi}\right\|}\left[\rho_{\Pi} g \frac{\left\|\mathbf{u}_{\Pi}\right\|^{2}}{\xi_{\Pi}}\right] .
$$

Direct air entrainment into the powder cloud is denoted

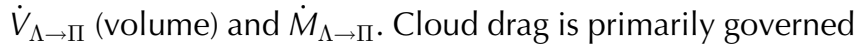
by turbulent air entrainment $\dot{M}_{\Lambda \rightarrow \Pi}$.

Two salient requirements for the formation of a powder cloud are (1) disperse flows that allow air intake and (2) the availability of ice dust to load the interstitial pore space with air tracers. Both processes are better facilitated with dry, cold snow. Another assumption is that the ingested air is accelerated to the speed of the dilute core. Air intake is therefore associated with an additional speed-dependent drag on the avalanche core. This drag is included in the model formulation.

\section{EVEREST ICE AVALANCHE, 25 APRIL 2015}

A mixed flowing/powder avalanche struck the Everest base camp (5250 ma.s.I.) on 25 April 2015. Severe earthquaking released large blocks of ice from a calving glacier located on Pumo Ri Peak (7156 ma.s.l.; Fig. 6). The air-blast of the powder avalanche killed 21 people. The destruction of the base camp was documented by eyewitnesses, facilitating a reconstruction of the event (Wilkinson, 2015).

To simulate this mixed flowing/powder avalanche we first obtained an accurate digital elevation model (DEM) as well as satellite imagery to identify the release location and volume. We purchased high spatial resolution optical satellite imagery before (8 April 2015, Pléiades satellite, $0.5 \mathrm{~m}$ spatial resolution, three different viewing angles) and after the avalanche release (30 April 2015 GeoEye1 satellite and 25 May 2015 WorldView2 satellite, $0.5 \mathrm{~m}$ spatial resolution). We used the Pléiades dataset to calculate a $2 \mathrm{~m}$ spatial resolution DEM applying state-of-the-art methods (Deilami and Hashim, 2011). In the upper, steep part of the Pumo Ri ridge, some snow-covered areas are saturated in the satellite imagery, resulting in holes within the DEM, which we filled by interpolation. By comparing the satellite imagery before and after the event we were able to identify a $70 \mathrm{~m} \times 50 \mathrm{~m}$ ice mass at $6150 \mathrm{~m}$ a.s.l. This release location is in agreement with eyewitness observations. The release mass was situated on steep terrain (slope angle $55^{\circ}$ ). The pre- and post-event satellite imagery was used to estimate the release height to be $\sim 10 \mathrm{~m}$, resulting in a total release volume of $53000 \mathrm{~m}^{3}$ of ice. For the calculations, we assumed the density of ice to be $800 \mathrm{~kg} \mathrm{~m}^{-3}$ at release; the deposition density of the ice avalanche core to be $\rho_{0}=$ $600 \mathrm{~kg} \mathrm{~m}^{-3}$. The volume of ice is not large in comparison to 


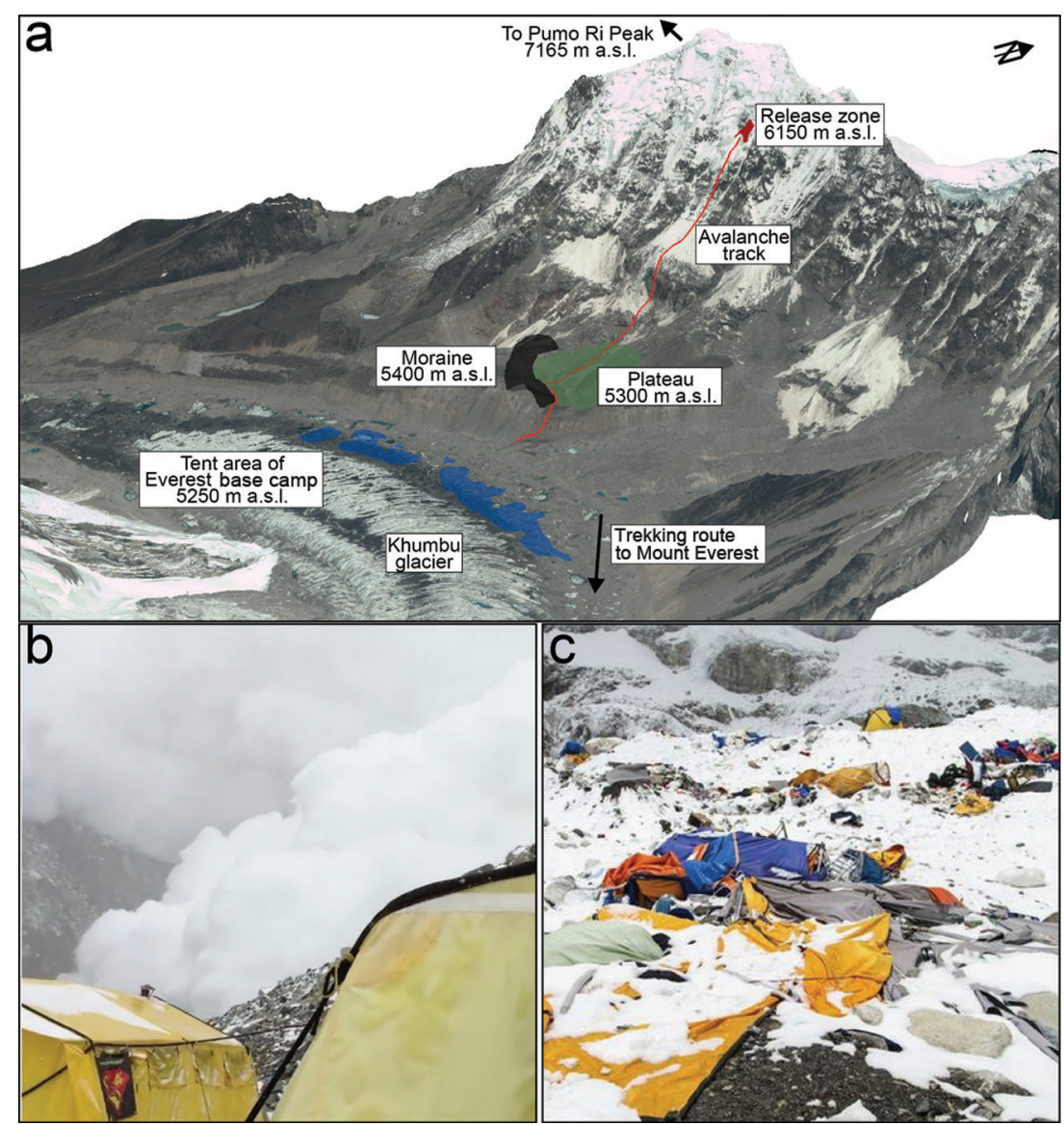

Fig. 6. Disposition of the ice avalanche that struck the Everest base camp after severe earthquaking on 25 April 2015. (a) The avalanche released at $6150 \mathrm{~m}$ a.s.l. and dropped only $900 \mathrm{~m}$. It struck a moraine at $5400 \mathrm{~m}$ a.s.l. and was deflected towards the upper base camp. (b) The powder cloud in the Everest base camp area. The cloud in the foreground is traveling in the direction of the lower base camp. The cloud in the background is overtopping the moraine. (c) Destruction from the air-blast. The estimated pressures are between 2 and $4 \mathrm{kPa}$.

other observed ice avalanches in the European Alps (Pralong and Funk, 2006).

To model the motion of the avalanche core we applied fluidization parameters similar to dry snow avalanches: $\alpha=0.10, \beta=0.80, \gamma=0.20$ and $R_{0}=4.0 \mathrm{~kJ} \mathrm{~m}^{3}$ (Buser and Bartelt, 2015). The activation energy $R_{0}$ was doubled to account for the fact that the density of the ice blocks is approximately twice the density of snow granules. The production parameter $\alpha$ is slightly higher than values used for snow avalanches, suggesting (1) extremely rough terrain and (2) possible further ground trembling after release, additionally helping to fluidize the avalanche core. We set $\mu_{0}=0.55$ and $\xi_{0}=1800 \mathrm{~m} \mathrm{~s}^{-2}$, similar to snow avalanches (Bartelt and others, 2012). For the powder cloud a drag coefficient of $\xi_{\Pi}=2000 \mathrm{~m} \mathrm{~s}^{-2}$ was applied. The embryonic density of the powder cloud we took to be $\rho_{\Pi_{0}}=5 \mathrm{~kg} \mathrm{~m}^{-3}$.

The simulations reveal that after the release the mass fell $350 \mathrm{~m}$ vertically and accelerated rapidly to a speed of $60 \mathrm{~m} \mathrm{~s}^{-1}$ (Fig. 7a). It then fell over a steep $200 \mathrm{~m}$ high cliff, reaching its terminal velocity of $75 \mathrm{~m} \mathrm{~s}^{-1}$ (Fig. 8a). The ice mass clearly fluidized in the steep and rough terrain of the Pumo Ri ridge. The calculated density of the core was at times lower than $200 \mathrm{~kg} \mathrm{~m}^{-3}$, indicating the intake and acceleration of large volumes of air. In the steep terrain above the plateau, the calculations reveal that the avalanche core was $>15 \mathrm{~m}$ high. The avalanche core crossed a long $600 \mathrm{~m}$ plateau where it slammed into a $100 \mathrm{~m}$ high moraine (Figs 7 and 9). This terrain feature deflected and slowed down the avalanche core from $70 \mathrm{~m} \mathrm{~s}^{-1}$ to $20 \mathrm{~m} \mathrm{~s}^{-1}$. With the assumption of a uniform core density, the model predicts ice particles could be thrown to heights higher than $50 \mathrm{~m}$ at impact.

The moraine was also high enough to block the motion of the powder cloud. Video recordings show only a slight overtopping of the moraine (Fig. 6b). To simulate the braking effect of the moraine it was therefore necessary to introduce no-flux boundary conditions for the cloud along the moraine ridge. Two-layer models are computationally efficient (the simulations using a $6 \mathrm{~m} \times 6 \mathrm{~m}$ grid resolution required $\sim 30 \mathrm{~min}$ on a PC) but cannot model the blocking or deflecting effect of terrain features larger than the height of the powder cloud. These can be effectively treated as noflux boundary conditions. Simulation scenarios without this moraine reveal that the destruction at the lower part of the Everest base camp would have been much more severe, certainly causing many more fatalities.

The moraine did not stop the avalanche completely. The avalanche reached the end of the plateau, fell an additional $100 \mathrm{~m}$ vertically and impacted the rough, undulated surface of Khumbu Glacier (Figs 7 and 9). Here the core deposited, which can be verified with the post-event satellite imagery. The extents of the observed and simulated deposits are in very good agreement (Fig. 7a and b). Most of the ice mass did not reach the valley but was deposited in the upper plateau. The deposition field in the upper plateau was $350 \mathrm{~m}$ long and $100 \mathrm{~m}$ wide; the maximum deposition height was $\sim 4 \mathrm{~m}$. The part of the powder cloud not stopped by the 


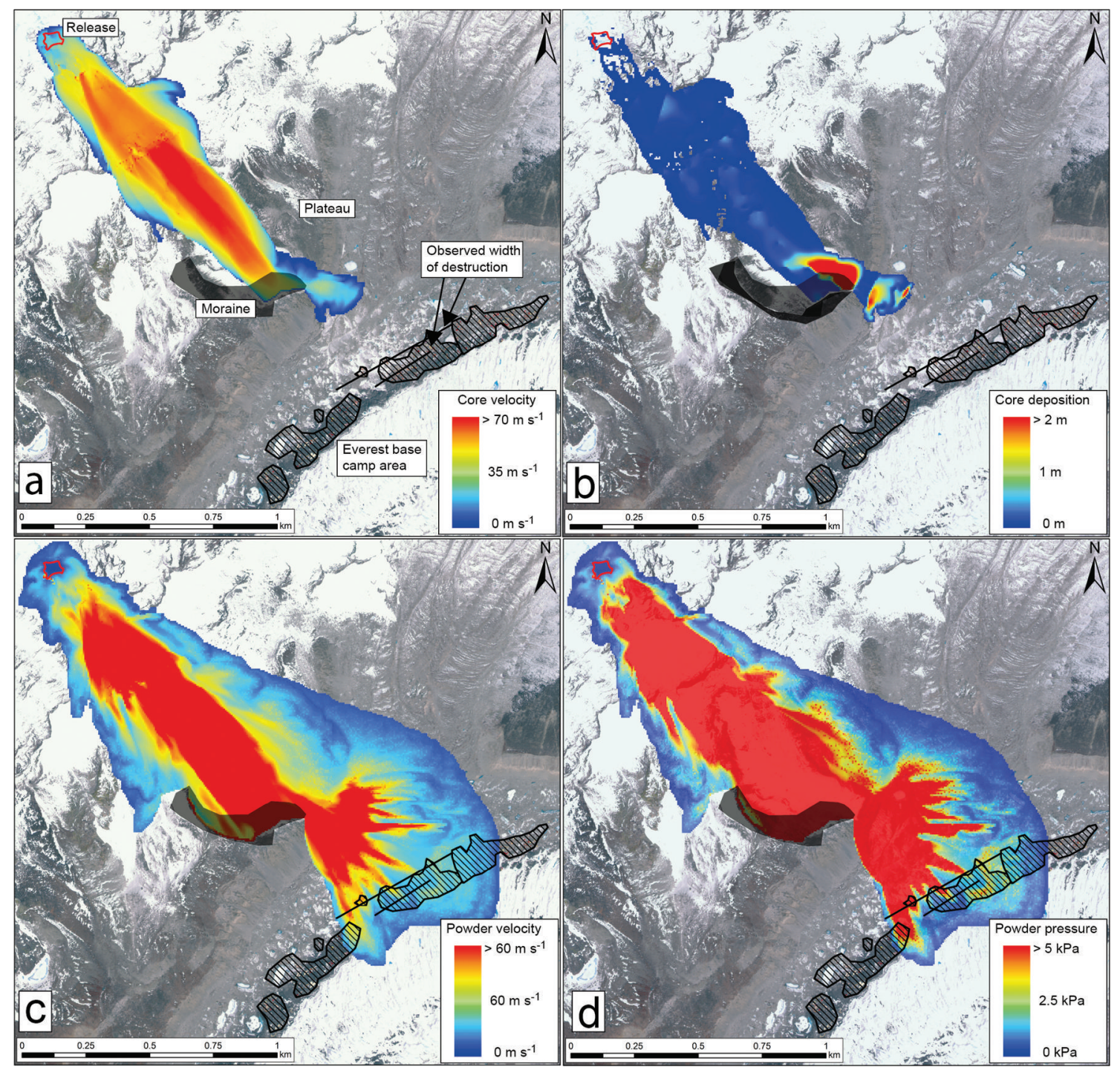

Fig. 7. Results of the model calculations. (a) Calculated velocities of the avalanche core. The core reached velocities approaching $70 \mathrm{~m} \mathrm{~s}^{-1}$. (b) Calculated deposition field of the avalanche core. Ice deposits of $>2 \mathrm{~m}$ with density $600 \mathrm{~kg} \mathrm{~m}^{-3}$ came to rest on the plateau. The deposition field below the moraine is in good agreement with post-event satelite images. (c) Calculated powder cloud velocities. The powder cloud was travelling with mean velocities $>60 \mathrm{~m} \mathrm{~s}^{-1}$ as it approached the base camp. (d) The calculated avalanche pressure field. Pressures of $>3 \mathrm{kPa}$ are found in parts of the base camp. The width of the powder cloud is in good agreement with eyewitness reports.

moraine, now decoupled independent from the decelerating core, continued its motion in the direction of the upper base camp, spreading radially with mean velocities between 30 and $40 \mathrm{~m} \mathrm{~s}^{-1}$ across Khumbu Glacier (Fig. 9). The height of the cloud exceeded $100 \mathrm{~m}$ as observed in the eyewitness videos (Fig. 6); the total volume of the powder cloud was calculated to be $>100 \times 10^{6} \mathrm{~m}^{3}$.

Climbers located at the upper Everest base camp were struck by a fast-moving powder cloud with peak densities of $3 \mathrm{~kg} \mathrm{~m}^{-3}$. The pressure map (Fig. $7 \mathrm{~d}$ ) depicts the total inundated area; the region in red where the air-blast pressures exceed $3 \mathrm{kPa}$. The calculated areas with pressures greater than $3 \mathrm{kPa}$ are also in good agreement with eyewitness sketches of the base camp damage and the location of the victims (Fig. 8b). We are aware of the drawbacks of a two-layer model which provides only the mean velocities and mean densities of the core and the cloud. However, for practical application it appears to be sufficient. The model presented here can be further developed to include the z-dependent profiles of velocity and density and therefore provide more accurate pressure results.

\section{CONCLUSIONS}

The formation of the powder cloud is divided into two steps: (1) air intake into the core and mixture with the ice dust tracers, $\dot{V}_{\Lambda \rightarrow \Phi}$, and (2) air blow-out $\dot{V}_{\Phi \rightarrow \Pi \text {. The model }}$ therefore divides the formation process into two discrete and sequential phases. However, the process occurs simultaneously, but at different locations in the avalanche flow. The slope-perpendicular expansion and compression of the avalanche core leads to intermittent blow-outs providing powder snow avalanches with their familiar plume structures with lobe (blow-out) and cleft (no blow-out) features. At the time of formation, additional air is entrained directly into the cloud $\dot{V}_{\Lambda \rightarrow \Pi}$ as the plumes rise (Davidson, 2004). This model assumes a close relation between the flowing core $\Phi$, the powder cloud $\Pi$ and the ambient air $\Lambda$.

The air-intake phase $\dot{V}_{\Lambda \rightarrow \Phi}$ requires a model to describe the expansion of the avalanche core. The core of an avalanche cannot be considered as a rigid solid block or isochoric body. The interaction of particles with the basal boundary creates a dispersive pressure which is responsible 

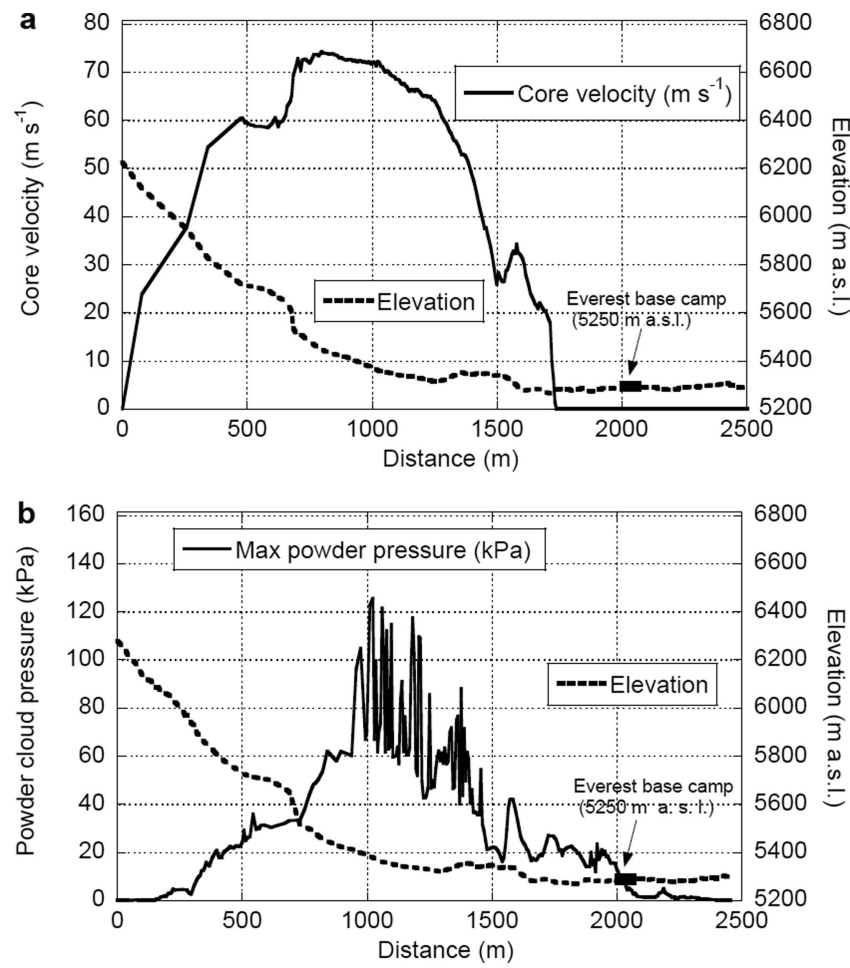

Fig. 8. Simulation results along track profile. (a) Maximum calculated velocity of the avalanche core. The core stops $\sim 250 \mathrm{~m}$ before the base camp. (b) Maximum calculated impact pressures. The impact pressures at the base camp are between 1 and $4 \mathrm{kPa}$. The pressures are largest on the plateau above the base camp where the avalanche core is travelling at the highest speed.

for changes in the location of the center-of-mass of the particle ensemble. Expansion of the core reduces its density by air intake. To create the dispersive pressure, particles must have a slope-perpendicular velocity component and therefore some kinetic energy that is not included in the slope-parallel direction. The effect of the dispersive pressure is to change the location of the particles and therefore the configurational energy of the core. The initial air intake into the core is therefore related to the total free energy, both the free kinetic energy $\left(R_{K}\right)$ and free configurational energy $\left(R_{V}\right)$. Because the source of the dispersive pressure is the interaction of particles with the boundary, the production of $R_{V}$ must be some part of the total production of free energy $\dot{P}$. Particle interactions free ice dust sufficient to load the enclosed air with tracers. At this stage, the cloud exists within the core. The core $(\Phi)$ and powder cloud (П) layers are together. They are combined in a furious ensemble of snow particles and ice dust. The air-intake phase is included in the model equations.

The blow-out phase begins as heavier particles settle, displacing the enclosed air. Because the avalanche is moving forward, the easiest escape for the ice dust mixture is upwards. Segregation of particles divides the avalanche into two layers, the core $\Phi$ and cloud $\Pi$. The blow-out phase requires the loss of potential energy $R_{V}$. Losses will occur when the decay of total free energy $R$ overcomes the production. The free energy parameters $\alpha$ (production) and $\beta$ (decay) therefore control the collapse and the blow-out process. We can imagine other processes that lead to the compression of the core, including streamwise velocity gradients (passive flow states) or terrain changes. Large plumes are often created during the sudden transition from steep to flat slopes. Such effects will be introduced in future models. At present, only centripetal pressures influence the blow-out process.

Once created, the cloud moves independently of the core. The momentum acquired in the core serves as an initial condition. Equations for the momentum of the core and the momentum of the powder cloud are therefore necessary and included in the model formulation. We assume the core provides the cloud with sufficient momentum to be destructive, unlike non-Boussinesq powder avalanche mod-

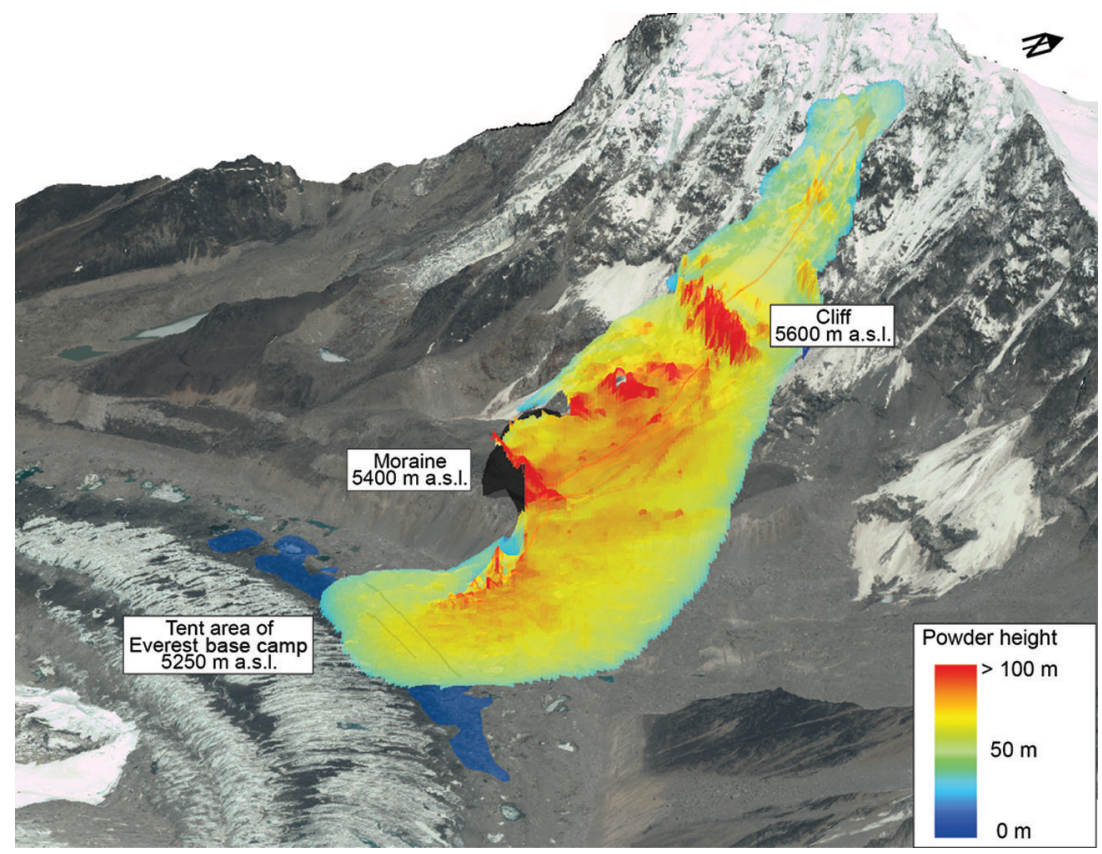

Fig. 9. Three-dimensional representation of extent of powder avalanche. The height of the clouds was largest after the avalanche fell over a $100 \mathrm{~m}$ cliff at $5600 \mathrm{~m}$ a.s.l. Cloud heights at the moraine were also large and slightly overtopped the $100 \mathrm{~m}$ high moraine. Cloud heights at the base camp were $>50 \mathrm{~m}$. The width of the cloud at the base camp is in good agreement with eyewitness accounts. 
els in which the driving force of the cloud arises only from the density difference between the ice particles and air (Fukushima and Parker, 1990; Ancey, 2004; Rastello and Hopfinger, 2004; Turnbull and McElwaine, 2007; Turnbull and others, 2007; Carroll and others, 2013). We assume the air and ice particles (tracers) are moving with the same velocity; the density of the particles alone cannot overcome Stokes drag. The velocity attenuation of the cloud, containing moving air laden with ice dust, is given by aerodynamic drag. This is a dissipative process, but not a severe one as evidenced by the long propagation distances of mixed flowing/powder snow and ice avalanches.

\section{ACKNOWLEDGEMENTS}

We sincerely thank Kolumban Hutter for his involvement in this paper, especially for long discussions of ideas concerning configurational energy and thermodynamics that first appeared in Luca and others (2004). Kolumban Hutter thanks the directorate of WSL-SLF for support.

\section{REFERENCES}

Ancey C (2004) Powder snow avalanches: approximation as nonBoussinesq clouds with a Richardson number-dependent entrainment function. J. Geophys. Res., 109, F01005 (doi: 10.1029/2003JF000052)

Bagnold RA (1954) Experiments on a gravity-free dispersion of large solid spheres in a Newtonian fluid under shear. Proc. R. Soc. London, Ser. A, 225(1160), 49-63

Bartelt P, Buser O and Platzer K (2006) Fluctuation-dissipation relations for granular snow avalanches. J. Glaciol., 52(179), 631-643 (doi: 10.3189/172756506781828476)

Bartelt P, Bühler O, Buser O, Christen M and Meier L (2012) Modeling mass-dependent flow regime transitions to predict the stopping and depositional behaviour of snow avalanches. J. Geophys. Res., 117, F01015 (doi: 10.1029/2010JF001957)

Bartelt P, Bühler Y, Buser O and Ginzler C (2013) Plume formation in powder snow avalanches: measurements and modelling of snow avalanche speeds. Proceedings of the International Snow Science Workshop, Grenoble - Chamonix Mont-Blanc, 2013. International Snow Science Workshop

Bozhinskiy AN and Losev KS (1998) The fundamentals of avalanche science. Eidg. Inst. Schnee- Lawinenforsch. 55

Bozhinskiy AN and Sukhanov LA (1998) Physical modelling of avalanches using an aerosol cloud of powder materials. Ann. Glaciol., 26, 242-246.

Buser $\mathrm{O}$ and Bartelt $\mathrm{P}$ (2009) Production and decay of random kinetic energy in granular snow avalanches. J. Glaciol., 55(189), 3-12 (doi: 10.3189/002214309788608859)

Buser O and Bartelt P (2011) Dispersive pressure and density variations in snow avalanches. J. Glaciol., 57(205), 857-860 (doi: 10.3189/002214311798043870)

Buser $\mathrm{O}$ and Bartelt P (2015) An energy-based method to calculate streamwise density variations in snow avalanches. J. Glaciol., 61(227), 563-575 (doi: 10.3189/2015JoG14J054)
Carroll C, Louge M and Turnbull B (2013) Frontal dynamics of powder snow avalanches. J. Geophys. Res., 118(2), 913-924 (doi: 10.1002/jgrf.200068)

CBS News (2015) 'Impossible' to find all dead in crushed Nepal village. 5 May http://www.cbsnews.com/news/nepal-earthquake-mudslide-buried-langtang-village-killed-foreign-trekkers/ [accessed 15 May 2015]

Christen M, Kowalski J and Bartelt P (2010) RAMMS: numerical simulation of dense snow avalanches in three-dimensional terrain. Cold Reg. Sci. Technol., 63(1-2), 1-14 (doi: 10.1016/ j.coldregions.2010.04.005)

Davidson PA (2004) Turbulence: an introduction for scientists and engineers. Oxford University Press, Oxford

Deilami K and Hashim M (2011) Very high resolution optical satellites for DEM generation: a review. Eur. J. Sci. Res., 49(4), 542-554

Fukushima Y and Parker G (1990) Numerical simulation of powder snow avalanches. J. Glaciol., 36(123), 229-237

Grigoryan S, Urubayev N and Nekrasov I (1982) Experimental investigation of an avalanche air blast. Data Glaciol. Stud., 44, 87-93 [in Russian]

Jenkins M (2015) Historic Tragedy on Everest, With 13 Sherpas Dead in Avalanche. National Geographic News http://news. nationalgeographic.com/news/2014/04/140418-everest-avalanche-sherpa-killed-mountain/ [accessed 15 May 2015]

Luca I, Fang C and Hutter K (2004) A thermodynamic model of turbulent motions in a granular material. Contin. Mech. Thermodyn., 16(4), 363-390 (doi: 10.1007/s00161-003-0163-z)

Nazarov AN (1990) Mathematical modeling of a snow powder avalanche in the framework of the equations of two-layer shallow water. [transl. from Isvestiya Akademii Nauk SSSR, Mekanika Zhidkosti i Gaza, No. 1, 84-90]

Platzer K, Bartelt P and Kern M (2007) Measurements of dense snow avalanche basal shear to normal stress ratios (S/N). Geophys. Res. Lett., 34(7), L07501 (doi: 10.1029/2006GL028670)

Pralong A and Funk M (2006) On the instability of avalanching glaciers. J. Glaciol., 52(176), 31-48 (doi: 10.3189/ 172756506781828980)

Rastello M and Hopfinger E (2004) Sediment-entraining suspension clouds: a model of powder-snow avalanches. J. Fluid Mech., 509, 181-206

Sukhanov G (1982) The mechanism of avalanche air blast formation as derived from field measurements. Data Glaciol Stud., 44, 94-98 [in Russian]

Sukhanov G and Kholobayev (1982) Variability of avalanche air blast in time and space. Data Glaciol Stud., 44, 98-105 [in Russian]

Turnbull B and McElwaine J (2007) A comparison of powder-snow avalanches at Vallée de la Sionne, Switzerland, with plume theories. J. Glaciol., 53(180), 30-40 (doi: 10.3189/ 172756507781833938)

Turnbull B, McElwaine J and Ancey C (2007) KulikovskySveshnikova-Begin model of powder snow avalanches: development and application. J. Geophys. Res., 112, F01004 (doi: 10.1029/2006JF000489)

Wilkinson F (2015) Survivors and Dead Evacuated from Everest Base Camp. National Geographic News www.news. nationalgeographic.com/2015/04/150426-everest-earthquakebasecamp-nepal-himalaya-climbing-sherpas/ [accessed 14 May 2015] 